\title{
On Three Generalizations of Contraction
}

\author{
Eduardo D. Sontag and Michael Margaliot and Tamir Tuller
}

\begin{abstract}
We introduce three forms of generalized contraction (GC). Roughly speaking, these are motivated by allowing contraction to take place after small transients in time and/or amplitude. Indeed, contraction is usually used to prove asymptotic properties, like convergence to an attractor or entrainment to a periodic excitation, and allowing initial transients does not affect this asymptotic behavior.

We provide sufficient conditions for GC, and demonstrate their usefulness using examples of systems that are not contractive, with respect to any norm, yet are GC.
\end{abstract}

\section{INTRODUCTION}

A dynamical system is called contractive if any two trajectories converge to one other at an exponential rate. This implies many desirable properties including convergence to an attractor (if it exists), and entrainment to periodic excitations [1], [2].

Contraction theory is a powerful tool for analyzing nonlinear dynamical systems, with applications in control theory [3], observer design [4], synchronization of coupled oscillators [5], and more. Recent extensions include: the notion of partial contraction [6], analyzing a network of interacting contractive elements [7], a Lyapunov-like characterization of incremental stability [8], and a LaSalle-type principle for contractive systems [9]. A contractive system with added diffusion terms or random noise still satisfies certain asymptotic properties [10], [11]. In this respect, contraction is a robust property.

In this paper, we introduce three forms of generalized contraction (GC). These are motivated by requiring contraction to take place only after arbitrarily small transients in time and/or amplitude. Indeed, contraction is usually used to prove asymptotic properties, and thus allowing (arbitrarily small) transients seems reasonable. We demonstrate the usefulness of these generalizations by showing several examples of systems that are not contractive with respect to any norm, yet are a GC.

The remainder of this paper is organized as follows. Section \ provides a brief overview of contraction theory. Section [II] describes our main results. The proofs of these results are detailed in Section VI] Section IV] demonstrates the results using a simple model of a biochemical control system.

EDS (sontag@math.rutgers.edu) is with the Dept. of Mathematics and the Cancer Institute of New Jersey, Rutgers University, Piscataway, NJ 08854, USA; MM (michaelm@eng.tau.ac.il) is with the School of Electrical Engineering-Systems and the Sagol School of Neuroscience, Tel Aviv University, Israel 69978; TT (tamirtul@ post.tau.ac.il) is with the Dept. of Biomedical Engineering and the Sagol School of Neuroscience, Tel-Aviv University, Tel-Aviv 69978, Israel. EDS's work is supported in part by grants NIH 1R01GM086881 and 1R01GM100473, and AFOSR FA955011-1-0247. The research of MM is partly supported by the ISF.

\section{CONTRACTION Theory}

We begin with a brief review of some ideas from contraction theory. For more details, including the historic development of contraction theory, and the relation to other notions, see e.g. [12], [13], [14].

Consider the time-varying system

$$
\dot{x}=f(t, x),
$$

evolving on a convex set $\Omega \subset \mathbb{R}^{n}$. We assume that $f(t, x)$ is differentiable with respect to $x$, and that both $f(t, x)$ and $J(t, x):=\frac{\partial f}{\partial x}(t, x)$ are continuous in $(t, x)$. Let $x\left(t, t_{0}, x_{0}\right)$ denote the solution of (1) at time $t \geq t_{0}$ with $x\left(t_{0}\right)=x_{0}$ (for the sake of simplicity, we assume from here on that $x\left(t, t_{0}, x_{0}\right)$ exists and is unique for all $t \geq t_{0} \geq 0$ and all $x_{0} \in \Omega$ ).

Recall that (1) is called contractive [1] on $\Omega$ with respect to a norm $|\cdot|: \mathbb{R}^{n} \rightarrow \mathbb{R}_{+}$if there exists $c>0$ such that

$$
\left|x\left(t_{2}, t_{1}, a\right)-x\left(t_{2}, t_{1}, b\right)\right| \leq \exp \left(-\left(t_{2}-t_{1}\right) c\right)|a-b|
$$

for all $t_{2} \geq t_{1} \geq 0$ and all $a, b \in \Omega$.

In other words, any two trajectories contract to one another at an exponential rate. This implies in particular that the initial condition is "forgotten".

Recall that a vector norm $|\cdot|: \mathbb{R}^{n} \rightarrow \mathbb{R}_{+}$induces a matrix measure $\mu: \mathbb{R}^{n \times n} \rightarrow \mathbb{R}$ defined by $\mu(A):=\lim _{\epsilon \downarrow 0} \frac{1}{\epsilon}(\| I+$ $\epsilon A \|-1$ ), where $\|\cdot\|: \mathbb{R}^{n \times n} \rightarrow \mathbb{R}_{+}$is the matrix norm induced by $|\cdot|$. A standard approach for proving contraction is based on bounding some matrix measure of the Jacobian $J$. (This is in fact a particular case of using a Lyapunov-Finsler function to prove contraction [9]).

Theorem 1 [2] If there exists a vector norm $|\cdot|$ and $c>$ 0 such that the induced matrix measure $\mu: \mathbb{R}^{n \times n} \rightarrow \mathbb{R}$ satisfies

$$
\mu(J(t, x)) \leq-c,
$$

for all $t_{2} \geq t_{1} \geq 0$ and all $x \in \Omega$ then (2) holds.

One important implication of contraction is entrainment to a periodic excitation. Recall that $f: \mathbb{R}_{+} \times \Omega \rightarrow \mathbb{R}$ is called T-periodic if

$$
f(t, x)=f(t+T, x)
$$

for all $t \geq 0$ and all $x \in \Omega$.

Theorem 2 [2] If (1) is contractive and $f$ is T-periodic then there exists a unique periodic solution $\alpha:[0, \infty] \rightarrow \Omega$ of (1), of period $T$, and

$$
\lim _{t \rightarrow \infty}|x(t, 0, a)-\alpha(t)|=0, \text { for all } a \in \Omega .
$$


In other words, every trajectory $x(t, 0, a)$ converges to the unique periodic solution. Entrainment is important in various applications ranging from biological systems [15], [2] to the stability of the power grid [16].

The next section presents our main results. All the proofs are placed in Section VI

\section{MAin RESUlts}

We begin by defining three generalizations of contraction.

Definition 1 The time-varying system (1) is said to be:

- $a$ contraction after a small overshoot and short transient (SOST) on $\Omega$ with respect to a norm $|\cdot|: \mathbb{R}^{n} \rightarrow \mathbb{R}_{+}$ if for each $\varepsilon>0$ and each $\tau>0$ there exists $\ell=$ $\ell(\tau, \varepsilon)>0$ such that

$$
\begin{aligned}
& \left|x\left(t_{2}+\tau, t_{1}, a\right)-x\left(t_{2}+\tau, t_{1}, b\right)\right| \\
& \leq(1+\varepsilon) \exp \left(-\left(t_{2}-t_{1}\right) \ell\right)|a-b|
\end{aligned}
$$

for all $t_{2} \geq t_{1} \geq 0$ and all $a, b \in \Omega$.

- a contraction after a small overshoot (SO) on $\Omega$ with respect to a norm $|\cdot|: \mathbb{R}^{n} \rightarrow \mathbb{R}_{+}$if for each $\varepsilon>0$ there exists $\ell=\ell(\varepsilon)>0$ such that

$$
\begin{aligned}
& \left|x\left(t_{2}, t_{1}, a\right)-x\left(t_{2}, t_{1}, b\right)\right| \\
& \quad \leq(1+\varepsilon) \exp \left(-\left(t_{2}-t_{1}\right) \ell\right)|a-b|
\end{aligned}
$$

for all $t_{2} \geq t_{1} \geq 0$ and all $a, b \in \Omega$.

- a contraction after a short transient (ST) on $\Omega$ with respect to a norm $|\cdot|: \mathbb{R}^{n} \rightarrow \mathbb{R}_{+}$if for each $\tau>0$ there exists $\ell=\ell(\tau)>0$ such that

$$
\begin{aligned}
\mid x\left(t_{2}+\tau,\right. & \left.t_{1}, a\right)-x\left(t_{2}+\tau, t_{1}, b\right) \mid \\
& \leq \exp \left(-\left(t_{2}-t_{1}\right) \ell\right)|a-b|
\end{aligned}
$$

for all $t_{2} \geq t_{1} \geq 0$ and all $a, b \in \Omega$.

It is clear that every contractive system is SOST, SO, and ST. Thus, all these notions are generalizations of contraction.

The motivation for these definitions stems from the fact that important applications of contraction are in proving asymptotic properties. For example, proving that an equilibrium point is globally attracting or that the state-variables entrain to a periodic excitation. These properties describe what happens as $t \rightarrow \infty$, and so it seems natural to generalize contraction in a way that allows initial transients in time and/or amplitude.

In particular, the definition of SOST is motivated by requiring contraction at an exponential rate, but only after an (arbitrarily small) time $\tau$, and with an (arbitrarily small) overshoot $(1+\varepsilon)$. However, as we will see below when the convergence rate $\ell$ may depend on $\varepsilon$ a somewhat richer behavior may occur. The definition of SO is similar to that of SOST, yet now the convergence rate $\ell$ depends only on $\varepsilon$, and there is no time transient $\tau$ (i.e., $\tau=0$ ). In other words, SO is a uniform (in $\tau$ ) version of SOST. It is clear that SO implies SOST and we will see below that under a mild technical condition on (1) SO and SOST are equivalent.

The next example shows that ST is not equivalent to contraction. Recall that the error function is defined as $\operatorname{erf}(z):=$ $\frac{2}{\sqrt{\pi}} \int_{0}^{z} \exp \left(-s^{2}\right) \mathrm{d} s$.

Example 1 Consider the scalar time-varying system

$$
\dot{x}(t)=\left(\exp \left(-t^{2}\right)-1\right) x(t)
$$

evolving on $\Omega:=(-1,1)$. It is straightforward to show that this system is not contractive with respect to any norm (note that the Jacobian $J(t)=\exp \left(-t^{2}\right)-1$ satisfies $\left.J(0)=0\right)$. Yet, (7) is ST. To show this, pick $\tau>0$. Note that for all $t \geq$ $t_{1}, x\left(t, t_{1}, a\right)=f\left(t, t_{1}\right) a$, where

$$
f\left(t, t_{1}\right):=\exp \left(g(t)-g\left(t_{1}\right)\right),
$$

with

$$
g(t):=\frac{\sqrt{\pi}}{2} \operatorname{erf}(t)-t .
$$

Thus, for any norm $|\cdot|: \mathbb{R} \rightarrow \mathbb{R}_{+}$,

$$
\left|x\left(t_{2}, t_{1}, a\right)-x\left(t_{2}, t_{1}, b\right)\right|=f\left(t_{2}, t_{1}\right)|a-b|,
$$

We need to show that there exists $\ell_{1}=\ell_{1}(\tau)>0$ such that

$$
f\left(t_{2}+\tau, t_{1}\right) \leq \exp \left(-\left(t_{2}-t_{1}\right) \ell_{1}\right), \quad \text { for all } t_{2} \geq t_{1} \geq 0,
$$

or, equivalently, that

$$
a\left(z, t_{1}\right) \leq 0, \quad \text { for all } t_{1}, z \geq 0,
$$

where $z:=t_{2}-t_{1}$, and $a\left(z, t_{1}\right):=g\left(t_{1}+\tau+z\right)-g\left(t_{1}\right)+z \ell_{1}$. Since

$$
\dot{g}(t)=\exp \left(-t^{2}\right)-1
$$

we have

$$
\begin{aligned}
\frac{d}{d z} a\left(z, t_{1}\right) & =\exp \left(-\left(t_{1}+\tau+z\right)^{2}\right)-1+\ell_{1} \\
& \leq \exp \left(-\tau^{2}\right)-1+\ell_{1} .
\end{aligned}
$$

Taking $\ell_{1}:=\left(1-\exp \left(-\tau^{2}\right)\right) / 2>0$, yields $\frac{d}{d z} a\left(z, t_{1}\right)<0$ for all $t_{1}, z \geq 0$, so

$$
\begin{aligned}
a\left(z, t_{1}\right) & \leq a\left(0, t_{1}\right) \\
& =g\left(t_{1}+\tau\right)-g\left(t_{1}\right) \\
& \leq 0,
\end{aligned}
$$

where the last inequality follows from (11). We conclude that (10) indeed holds, so (7) is ST.

For time-invariant systems evolving on a compact set it is possible to give a simple sufficient condition for ST. Let $\operatorname{Int}(S)$ denote the interior of a set $S$. We require the following definitions.

Definition 2 We say that (1) is non expansive (NE) with respect to the norm $|\cdot|$ if for all $a, b \in \Omega$ and all $s_{2}>s_{1} \geq 0$

$$
\left|x\left(s_{2}, s_{1}, a\right)-x\left(s_{2}, s_{1}, b\right)\right| \leq|a-b| .
$$

We say that (1) is weakly contractive (WC) if (12) holds with $\leq$ replaced by $<$. 
Definition 3 The time-invariant system

$$
\dot{x}=f(x),
$$

evolving on a compact and convex set $\Omega \subset \mathbb{R}^{n}$, is said to be interior contractive (IC) if it satisfies the following properties:

(a) for every $x_{0} \in \partial \Omega, x\left(t, x_{0}\right) \notin \partial \Omega$ for all $t>0$.

(b) there exists a matrix measure $\mu: \mathbb{R}^{n \times n} \rightarrow \mathbb{R}$ such that

$$
\mu(J(x))<0, \quad \text { for all } x \in \operatorname{Int}(\Omega) .
$$

Note that conditions (a) and (b) do not necessarily imply contraction on $\Omega$, as it is possible that $\mu(J(x))=0$ for some $x \in \partial \Omega$. Yet, (14) does imply that (13) is non-expansive on $\Omega$.

Theorem 3 If the system (13) is IC then it is ST.

As noted above, the introduction of the GC forms is motivated by the idea that contraction is used to prove asymptotic results, so allowing initial transients should increase the class of systems that can be analyzed while still allowing to prove asymptotic results. The next result demonstrates this.

Corollary 1 Suppose that (13) is IC. Then (13) admits a unique equilibrium point $e \in \operatorname{Int}(\Omega)$, and $\lim _{t \rightarrow \infty} x(t, a)=$ $e$ for all $a \in \Omega$.

One may perhaps expect that we can generalize Theorem 3 to the time-varying case as well, that is, that if the timevarying system (11), evolving in a compact and convex set $\Omega \subset \mathbb{R}^{n}$, satisfies:

(a) for every $x_{0} \in \partial \Omega$ and every $t_{1} \geq 0$,

$$
x\left(t, t_{1}, x_{0}\right) \notin \partial \Omega, \text { for all } t>t_{1},
$$

(b) there exists a matrix measure $\mu: \mathbb{R}^{n \times n} \rightarrow \mathbb{R}$ such that

$$
\mu(J(t, x))<0, \text { for all } x \in \operatorname{Int}(\Omega), \text { and all } t \geq t_{1} \geq 0,
$$

then (1) is ST on $\Omega$. However, the next example shows that this is not so.

Example 2 Consider the scalar system

$$
\dot{x}(t)=-\frac{x(t)}{t+1},
$$

evolving in $\Omega:=[-1,1]$. The Jacobian is $J(t, x)=-(t+$ $1)^{-1}$, and properties (15) and (16) hold. Yet, this system is not SOST on $\Omega$ (and, therefore, it is clearly not ST on $\Omega$ ). Indeed, assume otherwise. Pick $\tau, \varepsilon>0$. Then there exists $\ell=\ell(\tau, \varepsilon)>0$ such that (4) holds. Since $x(t)=$ $(t+1)^{-1} x(0), E q$. (4) with the particular choice $t_{1}=0$ implies that

$$
\left(t_{2}+\tau+1\right)^{-1} \leq(1+\varepsilon) \exp \left(-\ell t_{2}\right), \quad \text { for all } t_{2} \geq 0,
$$

i.e.,

$$
\exp \left(\ell t_{2}\right) \leq(1+\varepsilon)\left(t_{2}+\tau+1\right), \quad \text { for all } t_{2} \geq 0,
$$

but this clearly cannot hold for $t_{2}>0$ sufficiently large.
To provide a sufficient condition for generalized contraction of the time-varying system (1), we require the following definition.

Definition 4 System (1) is said to be nested contractive (NC) on $\Omega$ with respect to a norm $|\cdot|$ if there exist convex sets $\Omega_{\zeta} \subseteq$ $\Omega$, and norms $|\cdot|_{\zeta}: \mathbb{R}^{n} \rightarrow \mathbb{R}_{+}$, where $\zeta \in(0,1 / 2]$, such that the following conditions hold.

- $\cup_{\zeta \in(0,1 / 2]} \Omega_{\zeta}=\Omega$, and

$$
\Omega_{\zeta_{1}} \subseteq \Omega_{\zeta_{2}}, \quad \text { for all } \zeta_{1} \geq \zeta_{2} .
$$

- For every $\tau>0$ there exists $\zeta=\zeta(\tau) \in(0,1 / 2]$, with $\zeta(\tau) \rightarrow 0$ as $\tau \rightarrow 0$, such that for every $a \in \Omega$ and every $t_{1} \geq 0$

$$
x\left(t, t_{1}, a\right) \in \Omega_{\zeta}, \quad \text { for all } t \geq t_{1}+\tau,
$$

and (11) is contractive on $\Omega_{\zeta}$ with respect to $|\cdot|_{\zeta}$.

- The norms $|\cdot|_{\zeta}$ converge to $|\cdot|$ as $\zeta \rightarrow 0$, i.e., for every $\zeta>0$ there exists $s=s(\zeta)>0$, with $s(\zeta) \rightarrow 0$ as $\zeta \rightarrow 0$, such that

$$
(1-s)|y| \leq|y|_{\zeta} \leq(1+s)|y|, \quad \text { for all } y \in \Omega .
$$

- System (11) is non-expanding with respect to $|\cdot|$ on $\Omega$.

Eq. (18) means that after an arbitrarily short time every trajectory enters and remains in a subset $\Omega_{\zeta}$ of the state space on which we have contraction with respect to $|\cdot|_{\zeta}$.

\section{Theorem 4 If the system (1) is NC then it is SOST.}

The next example demonstrates the usefulness of Theorem 4 by using it to prove that the system in Example 1 is SOST without using the explicit solution (8).

Example 3 Consider again the scalar time-varying system (7). Fix arbitrary $t_{1} \geq 0, a_{1} \in(-1,1)$, and rewrite (7) as

$$
\begin{array}{ll}
\dot{x}_{1}=\left(\exp \left(-x_{2}^{2}\right)-1\right) x_{1}, & x_{1}\left(t_{1}\right)=a_{1}, \\
\dot{x}_{2}=1, & x_{2}\left(t_{1}\right)=t_{1},
\end{array}
$$

evolving on $\Omega:=\left\{x \in \mathbb{R}^{2}: x_{1} \in(-1,1), x_{2} \geq t_{1}\right\}$. Note that any two feasible initial conditions $a, b \in \Omega$ for this systems satisfy $a_{2}=b_{2}=t_{1}$. The Jacobian of (19) is

$$
J(x)=\left[\begin{array}{cc}
-1+\exp \left(-x_{2}^{2}\right) & -2 x_{1} x_{2} \exp \left(-x_{2}^{2}\right) \\
0 & 0
\end{array}\right] .
$$

For any $\zeta \in(0,1 / 2]$, let

$$
\Omega_{\zeta}:=\left\{x \in \Omega: x_{1} \in(-1,1), x_{2} \geq t_{1}+\zeta\right\},
$$

and let $|\cdot|_{\zeta}:=|\cdot|_{1}$, that is, the $L_{1}$ norm. Note that (18) holds with $\zeta(\tau):=\min \{\tau, 1 / 2\}$, and that for every $x \in \Omega_{\zeta}$,

$$
\begin{aligned}
J_{11}(x) & =-1+\exp \left(-x_{2}^{2}\right) \\
& \leq-1+\exp \left(-\left(t_{1}+\zeta\right)^{2}\right) \\
& <-1+\exp \left(-\zeta^{2}\right) \\
& <0 .
\end{aligned}
$$


Let $d_{i}\left(t_{2}, t_{1}, a, b\right):=\left|x_{i}\left(t_{2}, t_{1}, a\right)-x_{i}\left(t_{2}, t_{1}, b\right)\right|, i=1,2$. Then

$$
\begin{aligned}
\left|x\left(t_{2}, t_{1}, a\right)-x\left(t_{2}, t_{1}, b\right)\right|_{1} & =d_{1}\left(t_{2}, t_{1}, a, b\right)+d_{2}\left(t_{2}, t_{1}, a, b\right) \\
& =d_{1}\left(t_{2}, t_{1}, a, b\right)+\left|t_{2}-t_{2}\right| \\
& =\left|x_{1}\left(t_{2}, t_{1}, a\right)-x_{1}\left(t_{2}, t_{1}, b\right)\right| .
\end{aligned}
$$

Combining this with (20) implies that all the conditions in Theorem 4 hold, so we conclude that (19) is SOST with respect to the $L_{1}$ norm.

The next section describes, using a specific mathematical model, one possible mechanism for ST. Namely, as we change the parameters in a contractive system, it may become ST when it hits the "verge" of contraction. For two vectors $a, b \in \mathbb{R}^{n}$, we write $a \geq b$ if $a_{i} \geq b_{i}$ for $i=1, \ldots, n$. A matrix $M \in \mathbb{R}^{n \times n}$ is called Metzler if $m_{i j} \geq 0$ for all $i \neq j$.

\section{AN APPLiCATION: A BIOCHEMICAL CONTROL CIRCUIT}

Consider the system

$$
\begin{aligned}
\dot{x}_{1} & =g\left(x_{n}\right)-\alpha_{1} x_{1}, \\
\dot{x}_{2} & =x_{1}-\alpha_{2} x_{2}, \\
\dot{x}_{3} & =x_{2}-\alpha_{3} x_{3}, \\
& \vdots \\
\dot{x}_{n} & =x_{n-1}-\alpha_{n} x_{n},
\end{aligned}
$$

where $\alpha_{i}>0$, and

$$
g(u):=\frac{1+u}{k+u}, \quad \text { with } k>1 .
$$

As explained in [17, Ch. 4] this may model a simple biochemical control circuit for protein synthesis in the cell. The $x_{i}$ s represent concentrations of various macro-molecules in the cell and therefore must be non-negative. It is straightforward to verify that $x(0) \in \mathbb{R}_{+}^{n}$ implies that $x(t) \in \mathbb{R}_{+}^{n}$ for all $t \geq 0$.

\section{Proposition 1 Let $\alpha:=\prod_{i=1}^{n} \alpha_{i}$. If}

$$
k-1<\alpha k^{2}
$$

then (21) is a contraction on $\mathbb{R}_{+}^{n}$. If $k-1=\alpha k^{2}$ then (21) is not a contraction, with respect to any norm, on $\mathbb{R}_{+}^{n}$, yet it is $S O$ on $\mathbb{R}_{+}^{n}$.

Note that for all $x \in \mathbb{R}_{+}^{n}$,

$$
g^{\prime}\left(x_{n}\right)=\frac{k-1}{\left(k+x_{n}\right)^{2}} \leq \frac{k-1}{k^{2}}=g^{\prime}(0) .
$$

Thus (22) implies that contraction holds if and only if the "total dissipation" $\alpha$ is strictly larger than $g^{\prime}(0)$.

Using the fact that $g(u)<1$ for all $u \geq 0$ it is straightforward to show that the set

$$
\Omega_{r}:=r\left(\left[0, \alpha_{1}^{-1}\right] \times\left[0,\left(\alpha_{1} \alpha_{2}\right)^{-1}\right] \times \cdots \times\left[0, \alpha^{-1}\right]\right)
$$

is an invariant set of the dynamics for all $r \geq 1$. Combining this with Prop. 1 implies that 21, with $k-1 \leq \alpha k^{2}$, admits a unique equilibrium point $e \in \Omega_{1}$ and that

$$
\lim _{t \rightarrow \infty} x(t, a)=e, \quad \text { for all } a \in \mathbb{R}_{+}^{n} .
$$

This property also follows from a more general result [17, Prop. 4.2.1] that is proved using the theory of irreducible cooperative dynamical systems. Yet the contraction approach leads to new insights. For example, it implies that the distance between trajectories can only decrease, and can also be used to prove entrainment to suitable generalizations of 21) that include periodically-varying inputs.

In the next section, we describe several more related notions and explore the relations between them.

\section{Additional Notions and Relations}

It is straightforward to show that each of the three generalizations of contraction in Definition 11 implies that (1) is NE. One may perhaps expect that any of the three generalizations of contraction in Definition 1 also implies WC. By taking $t_{1}=s_{1}, \tau=\left(s_{2}-s_{1}\right) / 2>0$, and $t_{2}=s_{1}+\tau$ in (6) it follows that ST does imply WC. However, the next example shows that SO does not imply WC.

\section{Example 4 Consider the scalar time-varying system}

$$
\dot{x}(t)= \begin{cases}0, & 0 \leq t \leq 1, \\ -2 x(t), & 1<t\end{cases}
$$

evolving on $\Omega:=(-1,1)$. Clearly, the trajectories of this system are not contracting for $t \in[0,1]$. Yet, we claim that this system is SO. To show this, pick $\varepsilon>0$. Let

$$
\ell:=\min \{\log (1+\varepsilon), 1\}
$$

Note that $\ell=\ell(\varepsilon)>0$. To show that (5) holds, we consider two cases.

Case 1. Suppose that $t_{1} \in[0,1]$. In this case, the solution of (24) is

$$
x\left(t, t_{1}, a\right)= \begin{cases}a, & t_{1} \leq t \leq 1 \\ \exp (-2(t-1)) a, & 1 \leq t\end{cases}
$$

Thus,

$$
\begin{aligned}
d: & =\left|x\left(t_{2}, t_{1}, a\right)-x\left(t_{2}, t_{1}, b\right)\right| \\
& = \begin{cases}|a-b|, & t_{1} \leq t_{2} \leq 1, \\
\exp \left(-2\left(t_{2}-1\right)\right)|a-b|, & 1 \leq t_{2} .\end{cases}
\end{aligned}
$$

Let $r:=(1+\varepsilon) \exp \left(-\ell\left(t_{2}-t_{1}\right)\right)|a-b|$. It follows from 25) that

$$
r \geq(1+\varepsilon)^{1-t_{2}+t_{1}}|a-b|
$$

so if $t_{2} \leq 1$ then clearly $d \leq r$.

Now suppose that $t_{2}>1$. If $1+\varepsilon \geq \exp (1)$ then it follows 
from (25) that $\ell=1$, so

$$
\begin{aligned}
r & =(1+\varepsilon) \exp \left(-\left(t_{2}-t_{1}\right)\right)|a-b| \\
& \geq \exp (1) \exp \left(-t_{2}\right)|a-b| \\
& \geq \exp (2) \exp \left(-2 t_{2}\right)|a-b| \\
& =d .
\end{aligned}
$$

If $1+\varepsilon<\exp (1)$ then it follows from (25) that $\ell=\log (1+\varepsilon)$, so

$$
\begin{aligned}
r & =(1+\varepsilon)^{1-t_{2}+t_{1}}|a-b| \\
& \geq(1+\varepsilon)^{1-t_{2}}|a-b| \\
& \geq \exp \left(1-t_{2}\right)|a-b| \\
& \geq \exp (2) \exp \left(-2 t_{2}\right)|a-b| \\
& =d .
\end{aligned}
$$

Summarizing, in Case 1 we always have $d \leq r$.

Case 2. Suppose that $t_{1}>1$. In this case, the solution of (24) is $x\left(t, t_{1}, a\right)=\exp \left(-2\left(t-t_{1}\right)\right) a$, so $d=\exp \left(-2\left(t_{2}-\right.\right.$ $\left.\left.t_{1}\right)\right)|a-b|$. Since $\ell \leq 1, d \leq(1+\varepsilon) \exp \left(-\ell\left(t_{2}-t_{1}\right)\right)|a-b|=$ $r$. Thus, in Case 2 we also have $d \leq r$, and this proves $S O$.

Summarizing, (24) is SO although its trajectories do not contract for $t \in[0,1]$. Clearly, for every fixed $T>0$ we can build a system that is SO although its trajectories do not contract for $t \in[0, T]$.

\section{A. contraction after a small overshoot and short transient}

The next result presents two conditions that are equivalent to SOST.

Lemma 1 The following conditions are equivalent.

1) System (1) is SOST on $\Omega$ with respect to some vector norm $|\cdot|_{v}: \mathbb{R}^{n} \rightarrow \mathbb{R}_{+}$.

2) For each $\tau>0$ there exists $\ell=\ell(\tau)>0$ such that

$$
\begin{aligned}
& \left|x\left(t_{2}+\tau, t_{1}, a\right)-x\left(t_{2}+\tau, t_{1}, b\right)\right|_{v} \\
& \leq(1+\tau) \exp \left(-\left(t_{2}-t_{1}\right) \ell\right)|a-b|_{v},
\end{aligned}
$$

for all $t_{2} \geq t_{1} \geq 0$ and all $a, b \in \Omega$.

3) For each $\varepsilon>0$ and each $\tau>0$ there exists $\ell_{1}=$ $\ell_{1}(\tau, \varepsilon)>0$ such that

$$
\left|x\left(t, t_{1}, a\right)-x\left(t, t_{1}, b\right)\right|_{v} \leq(1+\varepsilon) \exp \left(-\left(t-t_{1}\right) \ell_{1}\right)|a-b|_{v},
$$

for all $t \geq t_{1}+\tau \geq \tau$ and all $a, b \in \Omega$.

\section{B. contraction after a small overshoot}

A natural question is under what conditions SO and SOST are equivalent. To address this issue, we introduce the following definition.

Definition 5 We say that (1) is weakly expansive (WE) if for each $\delta>0$ there exists $\tau_{0}>0$ such that for all $a, b \in \Omega$ and all $t_{0} \geq 0$

$$
\left|x\left(t, t_{0}, a\right)-x\left(t, t_{0}, b\right)\right| \leq(1+\delta)|a-b|,
$$

for all $t \in\left[t_{0}, t_{0}+\tau_{0}\right]$.
Proposition 2 Suppose that (1) is WE. Then (1) is SOST if and only if it is SO.

Remark 1 Suppose that $f$ in (1) is Lipschitz globally in $\Omega$ uniformly in $t$, i.e. there exists $L>0$ such that

$$
|f(t, x)-f(t, y)| \leq L|x-y|, \quad \text { for all } x, y \in \Omega, t \geq t_{0} .
$$

Then by Gronwall's Lemma (see, e.g. [18, Appendix C])

$$
\left|x\left(t, t_{0}, a\right)-x\left(t, t_{0}, b\right)\right| \leq \exp \left(L\left(t-t_{0}\right)\right)|a-b|,
$$

for all $t \geq t_{0}$, and this implies that (29) holds for $\tau_{0}:=$ $\frac{1}{L} \log (1+\delta)>0$. In particular, if $\Omega$ is compact and $f$ is periodic in then WE holds under rather weak continuity arguments on $f$.

Fig. 1 summarizes the relations between the various contraction notions.

\section{PROOFS}

Proof of Theorem 3 .

We require the following result.

Lemma 2 If system (13) is IC then for each $\tau>0$ there is a $d>0$ such that

$$
\operatorname{dist}\left(x\left(t, x_{0}\right), \partial \Omega\right) \geq d,
$$

for all $x_{0} \in \Omega$ and all $t \geq \tau$.

Proof of Lemma 2. Pick $\tau>0$ and $x_{0} \in \Omega$. The assumption that $\Omega$ is invariant implies that $\operatorname{Int}(\Omega)$ is also an invariant set of (13) (see, e.g., [19, Lemma III.6]). Combining this with (a) implies that $x\left(t, x_{0}\right) \notin \partial \Omega$ for all $x_{0} \in \Omega$ and all $t>0$, so $e_{x_{0}}:=\operatorname{dist}\left(x\left(\tau, x_{0}\right), \partial \Omega\right)>0$. Thus, there exists a neighborhood $U_{x_{0}}$ of $x_{0}$, such that $\operatorname{dist}(x(\tau, y), \partial \Omega) \geq e_{x_{0}} / 2$ for all $y \in U_{x_{0}}$. Cover $\Omega$ by such $U_{x_{0}}$ sets. By compactness of $\Omega$, we can pick a finite subcover. Pick smallest $e$ in this subcover, and denote this by $d$. Then $d>0$ and we have that $\operatorname{dist}\left(x\left(\tau, x_{0}\right), \partial \Omega\right) \geq d$ for all $x_{0} \in \Omega$. Now, pick $t \geq \tau$ and $x_{0} \in \Omega$. Let $x_{1}:=x\left(t-\tau, x_{0}\right)$. Then:

$$
\operatorname{dist}\left(x\left(t, x_{0}\right), \partial \Omega\right)=\operatorname{dist}\left(x\left(\tau, x_{1}\right), \partial \Omega\right) \geq d,
$$

and this completes the proof of Lemma 2 ,

To prove Theorem 3 , pick $\tau>0$. Let $S_{\tau}:=\left\{x\left(t, x_{0}\right)\right.$ : $\left.t \geq \tau, x_{0} \in \Omega\right\}$. Lemma 2 implies that there exists a closed and convex set $D$ such that

$$
S_{\tau} \subseteq D \subset \operatorname{Int}(\Omega)
$$

(Note that since $\Omega$ is convex so is $\operatorname{Int}(\Omega)$ ). Let $c_{\tau}:=$ $\min _{x \in D} \mu(J(x))$. Then $c_{\tau}<0$. Thus, the system is contractive on $D$, and for all $a, b \in \Omega$ and all $t \geq 0$

$$
|x(\tau+t, a)-x(\tau+t, b)| \leq \exp \left(c_{\tau} t\right)|a-b|,
$$

where $|\cdot|$ is the vector norm corresponding to the matrix measure $\mu$. This establishes ST, and thus completes the proof of Theorem 3 . 


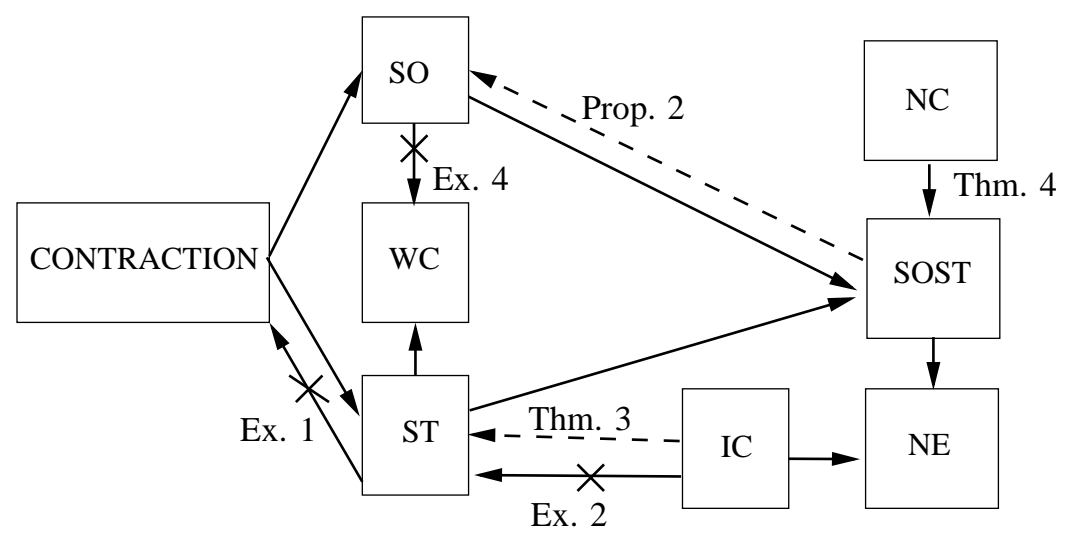

Fig. 1. Relations between various contraction notions. An arrow denotes implication; a crossed out arrow denotes that the implication is in general false; and a dashed arrow denotes an implication that holds under an additional condition. Some of the relations are immediate. Others follow from the results marked near the arrows.

\section{Proof of Corollary 1}

Since $\Omega$ is convex, compact, and invariant, it includes an equilibrium point $e$ of (13). By Theorem 3 the system is ST. Pick $a \in \Omega$ and $\tau>0$. Applying (6) with $b=e$ yields

$$
\left|x\left(t_{2}+\tau, t_{1}, a\right)-e\right| \leq \exp \left(-\left(t_{2}-t_{1}\right) \ell\right)|a-e|,
$$

for all $t_{2} \geq t_{1} \geq 0$, where $\ell>0$. This completes the proof. $\square$

\section{Proof of Theorem 4.}

Fix arbitrary $\varepsilon>0$ and $t_{1} \geq 0$. The function $\zeta=\zeta(\tau) \in$ $(0,1 / 2]$ is as in the statement of the Theorem. For each $\tau>$ 0 , let $c_{\zeta}>0$ be a contraction constant on $\Omega_{\zeta}$, where we write $\zeta=\zeta(\tau)$ here and in what follows. Take any $a, b \in \Omega$. By (18), $x\left(t, t_{1}, a\right), x\left(t, t_{1}, b\right) \in \Omega_{\zeta}$ for all $t \geq t_{1}+\tau$, so

$$
\begin{aligned}
\left|x\left(t, t_{1}, a\right)-x\left(t, t_{1}, b\right)\right|_{\zeta} \leq \exp \left(-c_{\zeta}\left(t-t_{1}-\tau\right)\right) \\
\times\left|x\left(t_{1}+\tau, t_{1}, a\right)-x\left(t_{1}+\tau, t_{1}, b\right)\right|_{\zeta}
\end{aligned}
$$

for all $t \geq t_{1}+\tau$. From the convergence property of norms in the Theorem statement, there exist $v_{\zeta}, w_{\zeta}>0$ such that

$$
|y| \leq v_{\zeta}|y|_{\zeta} \leq w_{\zeta} v_{\zeta}|y|, \quad \text { for all } y \in \Omega
$$

and $v_{\zeta} \rightarrow 1, w_{\zeta} \rightarrow 1$ as $\tau \rightarrow 0$. For $t \geq t_{1}+\tau$ let $p:=$ $t-t_{1}-\tau$. Then

$$
\begin{aligned}
& \left|x\left(t, t_{1}, a\right)-x\left(t, t_{1}, b\right)\right| \\
& \quad \leq v_{\zeta} \exp \left(-c_{\zeta} p\right)\left|x\left(t_{1}+\tau, t_{1}, a\right)-x\left(t_{1}+\tau, t_{1}, b\right)\right|_{\zeta} \\
& \quad \leq v_{\zeta} w_{\zeta} \exp \left(-c_{\zeta} p\right)\left|x\left(t_{1}+\tau, t_{1}, a\right)-x\left(t_{1}+\tau, t_{1}, b\right)\right| \\
& \quad \leq v_{\zeta} w_{\zeta} \exp \left(-c_{\zeta} p\right)|a-b|,
\end{aligned}
$$

where the last inequality follows from the fact that the system is non-expanding with respect to $|\cdot|$. Since $v_{\zeta} \rightarrow 1$, $w_{\zeta} \rightarrow 1$ as $\tau \rightarrow 0, v_{\zeta} w_{\zeta} \leq 1+\varepsilon$ for $\tau>0$ small enough. Summarizing, there exists $\tau_{m}=\tau_{m}(\varepsilon)>0$ such that for all $\tau \in\left[0, \tau_{m}\right]$

$$
\begin{aligned}
& \left|x\left(t+\tau, t_{1}, a\right)-x\left(t+\tau, t_{1}, b\right)\right| \\
& \quad \leq(1+\varepsilon) \exp \left(-c_{\zeta}\left(t-t_{1}\right)\right)|a-b|,
\end{aligned}
$$

for all $a, b \in \Omega$ and all $t \geq t_{1}$. Now pick $\tau>\tau_{m}$. For any $t \geq t_{1}$, let $s:=t+\tau-\tau_{m}$. Then

$$
\begin{aligned}
\mid x\left(t+\tau, t_{1}, a\right) & -x\left(t+\tau, t_{1}, b\right) \mid \\
& =\left|x\left(s+\tau_{m}, t_{1}, a\right)-x\left(s+\tau_{m}, t_{1}, b\right)\right| \\
\leq & (1+\varepsilon) \exp \left(-c_{\zeta}\left(s-t_{1}\right)\right)|a-b| \\
\leq & (1+\varepsilon) \exp \left(-c_{\zeta}\left(t-t_{1}\right)\right)|a-b|,
\end{aligned}
$$

and this completes the proof.

Proof of Proposition 1 .

We require the following result from [15].

Lemma 3 Consider a time-varying system

$$
\dot{x}=f(t, x)
$$

evolving on a subset of $X:=I_{1} \times I_{2} \times \ldots \times I_{n} \subseteq \mathbb{R}_{+}^{n}$, where each $I_{j}$ is an interval of the form $[0, a], a>0$, or $[0, \infty)$. Suppose that the time-dependent vector field $f=$ $\left[f_{1}, \ldots, f_{n}\right]^{\prime}$ has the following boundary-repelling property:

(BR) For each $\delta>0$ and each sufficiently small $\Delta>0$, there exists $K=K(\delta, \Delta)>0$ such that, for each $k=$ $1, \ldots, n$ and each $t \geq 0$, the condition

$$
x_{k} \leq \Delta \text { and } x_{i} \geq \delta, \quad \text { for every } 1 \leq i \leq k-1
$$

(for $k=1$, the condition is simply $x_{1} \leq \Delta$ )

implies that

$$
f_{k}(t, x) \geq K, \quad \text { for all } t \geq 0 .
$$

Then given any $\tau>0$ there exists $\varepsilon=\varepsilon(\tau)>0$, with $\varepsilon(\tau) \rightarrow$ 0 as $\tau \rightarrow 0$, such that, for every solution $x(t), t \geq 0$, it holds that $x(t) \geq \varepsilon$ for all $t \geq \tau$.

In other words, the conclusion is that after an arbitrarily short time every $x_{i}(t)$ is separated away from zero. 
To prove Proposition 1 note that the Jacobian of 21 is

$$
J(x)=\left[\begin{array}{cccccc}
-\alpha_{1} & 0 & 0 & \ldots & 0 & g^{\prime}\left(x_{n}\right) \\
1 & -\alpha_{2} & 0 & \ldots & 0 & 0 \\
0 & 1 & -\alpha_{3} & \ldots & 0 & 0 \\
& & \vdots & & & \\
0 & 0 & 0 & \ldots & 1 & -\alpha_{n}
\end{array}\right] .
$$

Thus, $J(x)$ is a Metzler matrix, so (21) is a monotone dynamical system [17]. It is well-known [20, Ch. 3] that the induced matrix measure corresponding to the $L_{1}$ vector norm is $\mu_{1}(A)=\max \left\{c_{1}(A), \ldots, c_{n}(A)\right\}$, where

$$
c_{j}(A):=A_{j j}+\sum_{\substack{1 \leq i \leq n \\ i \neq j}}\left|A_{i j}\right|
$$

i.e., the sum of the entries in column $j$ of $A$, with non diagonal elements replaced by their absolute values. Of course, if $A$ is Metzler then one can take $A_{i j}$ instead of $\left|A_{i j}\right|$ in (36). If $P$ is an invertible matrix, and $|\cdot|_{1, P}: \mathbb{R}^{n} \rightarrow \mathbb{R}_{+}$is the vector norm defined by $|z|_{1, P}:=|P z|_{1}$, then the induced matrix measure is $\mu_{1, P}(A):=\mu_{1}\left(P A P^{-1}\right)$. Let

$D_{\varepsilon}:=\operatorname{diag}\left(1, \alpha_{1}-\varepsilon,\left(\alpha_{1}-\varepsilon\right)\left(\alpha_{2}-\varepsilon\right), \ldots, \prod_{i=1}^{n-1}\left(\alpha_{i}-\varepsilon\right)\right)$, with $\varepsilon>0$ sufficiently small. Then

$D_{\varepsilon} J(x) D_{\varepsilon}^{-1}$

$$
=\left[\begin{array}{cccccc}
-\alpha_{1} & 0 & 0 & \ldots & 0 & \frac{g^{\prime}\left(x_{n}\right)}{\prod_{i=1}^{n-1}\left(\alpha_{i}-\varepsilon\right)} \\
\alpha_{1}-\varepsilon & -\alpha_{2} & 0 & \ldots & 0 & 0 \\
0 & \alpha_{2}-\varepsilon & 0 & \ldots & 0 & 0 \\
& & \vdots & & & \\
0 & 0 & 0 & \ldots & \alpha_{n-1}-\varepsilon & -\alpha_{n}
\end{array}\right],
$$

so

$$
\begin{aligned}
\mu_{1, D_{\varepsilon}}(J(x)) & =\max \left\{-\varepsilon, \frac{g^{\prime}\left(x_{n}\right)}{\prod_{i=1}^{n-1}\left(\alpha_{i}-\varepsilon\right)}-\alpha_{n}\right\} \\
& =\max \left\{-\varepsilon, \frac{g^{\prime}\left(x_{n}\right)-\alpha_{n} \prod_{i=1}^{n-1}\left(\alpha_{i}-\varepsilon\right)}{\prod_{i=1}^{n-1}\left(\alpha_{i}-\varepsilon\right)}\right\} .
\end{aligned}
$$

Suppose that $k-1<\alpha k^{2}$. Then for all $x \in \mathbb{R}_{+}^{n}$,

$$
g^{\prime}\left(x_{n}\right)=\frac{k-1}{\left(k+x_{n}\right)^{2}} \leq \frac{k-1}{k^{2}}<\alpha .
$$

Combining this with 37 implies that there exists a sufficiently small $\varepsilon>0$ such that $\mu_{1, D_{\varepsilon}}(J(x))<-\varepsilon / 2$ for all $x \in \mathbb{R}_{+}^{n}$, so the system is contractive on $\mathbb{R}_{+}^{n}$.

Now assume that $k-1=\alpha k^{2}$. By (35),

$$
\operatorname{det}(J(x))=(-1)^{n}\left(\alpha-g^{\prime}\left(x_{n}\right)\right),
$$

so for every $x \in \mathbb{R}_{+}^{n}$ with $x_{n}=0$, we have $\operatorname{det}(J(x))=$ $(-1)^{n}\left(\alpha-g^{\prime}(0)\right)=0$. This implies that the system is not contractive, with respect to any norm, on $\mathbb{R}_{+}^{n}$.
We now use Theorem 4 to prove that (21) is SOST. Note that since $g^{\prime}(u)=\frac{k-1}{(k+u)^{2}}$ and $k>1$,

$$
g\left(x_{n}\right) \geq g(0)=1 / k, \quad \text { for all } x \in \mathbb{R}_{+}^{n} .
$$

For $\zeta \in(0,1 / 2]$, let

$$
\Omega_{\zeta}:=\left\{x \in \mathbb{R}_{+}^{n}: x \geq \zeta\right\} .
$$

It is straightforward to verify that 21) satisfies condition (BR) in Lemma 3. Hence, for every $\tau>0$ there exists $\varepsilon(\tau)>0$ such that $x(t) \in \Omega_{\varepsilon}$ for all $t \geq \tau$. Then

$$
g^{\prime}\left(x_{n}\right)=\frac{k-1}{\left(k+x_{n}\right)^{2}} \leq \frac{k-1}{(k+\varepsilon)^{2}}<\frac{k-1}{k^{2}}=\alpha .
$$

We already showed that this implies that there exists a $\zeta>0$ and a norm $|\cdot|_{1, D_{\zeta}}$ such that (21) is contractive on $\Omega_{\varepsilon}$ with respect to this norm. When $\zeta=0$, 37 yields $\mu_{1, D_{0}}(J(x))=$ $\max \left\{0, \frac{g^{\prime}\left(x_{n}\right)-\alpha}{\alpha_{n-1} \ldots \alpha_{2} \alpha_{1}}\right\}$, and since $g^{\prime}\left(x_{n}\right)<\alpha, \mu_{1, D_{0}}(J(x)) \equiv$ 0 . Thus, 21) is NE with respect to $|\cdot|_{1, D_{0}}$. Summarizing, all the conditions in Theorem 4 hold, and thus the system is SOST. By Remark 1, this implies SO.

\section{Proof of Lemma 1}

If (1) is SOST then (27) holds for the particular case $\varepsilon=\tau$ in Definition 1. To prove the converse implication, assume that (27) holds. Pick $\hat{\tau}, \hat{\varepsilon}>0$. Let

$$
\tau:=\min \{\hat{\tau}, \hat{\varepsilon}\},
$$

and let $\ell=\ell(\tau)>0$. Pick $t \geq t_{1} \geq 0$, and let $t_{2}:=$ $t+\hat{\tau}-\tau \geq t_{1}$. Then

$$
\begin{aligned}
\mid x\left(t_{2}+\tau,\right. & \left.t_{1}, a\right)-\left.x\left(t_{2}+\tau, t_{1}, b\right)\right|_{v} \\
& \leq(1+\tau) \exp \left(-\left(t_{2}-t_{1}\right) \ell\right)|a-b|_{v} \\
& \leq(1+\hat{\varepsilon}) \exp \left(-\left(t-t_{1}\right) \ell\right)|a-b|_{v}
\end{aligned}
$$

where the last inequality follows from (38). Thus,

$$
\begin{aligned}
& \left|x\left(t+\hat{\tau}, t_{1}, a\right)-x\left(t+\hat{\tau}, t_{1}, b\right)\right|_{v} \\
& \leq(1+\hat{\varepsilon}) \exp \left(-\left(t-t_{1}\right) \ell\right)|a-b|_{v},
\end{aligned}
$$

and recalling that $\hat{\tau}, \hat{\varepsilon}>0$ were arbitrary, we conclude that Condition 2) in Lemma 1 implies SOST.

To prove that Condition 3) is equivalent to SOST, suppose that (28) holds. Then for any $t_{2} \geq t_{1}$,

$$
\begin{aligned}
\mid x\left(t_{2}+\tau, t_{1}, a\right)- & \left.x\left(t_{2}+\tau, t_{1}, b\right)\right|_{v} \\
& \leq(1+\varepsilon) \exp \left(-\left(t_{2}+\tau-t_{1}\right) \ell_{1}\right)|a-b|_{v} \\
& \leq(1+\varepsilon) \exp \left(-\left(t_{2}-t_{1}\right) \ell_{1}\right)|a-b|_{v},
\end{aligned}
$$

so we have SOST. Conversely, suppose that (1) is SOST. Pick any $\tau, \varepsilon>0$. Then there exists $\ell=\ell(\tau, \varepsilon / 2)>0$ such that for any $t \geq t_{1}+\tau$

$$
\begin{aligned}
\mid x\left(t, t_{1}, a\right)-x & \left.\left(t, t_{1}, b\right)\right|_{v} \\
& =\left|x\left(t-\tau+\tau, t_{1}, a\right)-x\left(t-\tau+\tau, t_{1}, b\right)\right|_{v} \\
& \leq(1+\varepsilon / 2) \exp \left(-\left(t-\tau-t_{1}\right) \ell\right)|a-b|_{v} .
\end{aligned}
$$


Thus, for any $c \in(0,1)$

$$
\begin{aligned}
\mid x\left(t, t_{1}, a\right)-x & \left.\left(t, t_{1}, b\right)\right|_{v} \\
\leq & (1+\varepsilon / 2) \exp (\tau c \ell) \exp \left(-\left(t-t_{1}\right) c \ell\right)|a-b|_{v} .
\end{aligned}
$$

Taking $c>0$ sufficiently small such that $(1+$ $\varepsilon / 2) \exp (\tau c \ell) \leq 1+\varepsilon$ implies that (28) holds for $\ell_{1}:=c \ell$. This completes the proof that (28) is equivalent to SOST.

Proof of Proposition 2

Suppose that (1) is SOST with respect to some norm $\mid$. $\left.\right|_{v}$. Pick $\varepsilon>0$. Since the system is WE, there exists $\tau_{0}=$ $\tau_{0}(\varepsilon)>0$ such that

$$
\left|x\left(t, t_{0}, a\right)-x\left(t, t_{0}, b\right)\right|_{v} \leq(1+\varepsilon / 2)|a-b|_{v},
$$

for all $t \in\left[t_{0}, t_{0}+\tau_{0}\right]$. Letting $\ell_{2}:=\frac{1}{\tau_{0}} \log \left(\frac{1+\varepsilon}{1+(\varepsilon / 2)}\right)$ yields

$$
\left|x\left(t, t_{0}, a\right)-x\left(t, t_{0}, b\right)\right|_{v} \leq(1+\varepsilon) \exp \left(-\left(t-t_{0}\right) \ell_{2}\right)|a-b|_{v},
$$

for all $t \in\left[t_{0}, t_{0}+\tau_{0}\right]$. By item 3 in Lemma 11 there exists $\ell_{1}=\ell_{1}\left(\tau_{0}, \varepsilon\right)>0$ such that

$\left|x\left(t, t_{0}, a\right)-x\left(t, t_{0}, b\right)\right|_{v} \leq(1+\varepsilon) \exp \left(-\left(t-t_{0}\right) \ell_{1}\right)|a-b|_{v}$,

for all $t \geq t_{0}+\tau_{0}$. Combining this with (39) yields

$\left|x\left(t, t_{0}, a\right)-x\left(t, t_{0}, b\right)\right|_{v} \leq(1+\varepsilon) \exp \left(-\left(t-t_{0}\right) \ell\right)|a-b|_{v}$,

for all $t \geq t_{0}$, where $\ell:=\min \left\{\ell_{1}, \ell_{2}\right\}>0$. This proves SO.

\section{CONCLUSIONS}

Contraction theory has proved useful for studying numerous dynamical systems. Contraction implies several desirable asymptotic properties such as convergence to a unique attractor (if it exists) and entrainment to periodic excitation. However, proving contraction is in many cases non-trivial.

In this paper, we introduced three generalizations of contraction. These are motivated by allowing contraction to take place after an arbitrarily small transient in time and/or amplitude. We provided conditions guaranteeing that these forms of GC hold, and demonstrated their usefulness by using them to analyze systems that are not contractive, with respect to any norm, yet are a GC.

We note in passing that our original motivation for generalizating contraction was to prove entrainment in a model for translation-elongation called the ribosome flow model (RFM) [21] (see also [22], [23], [24], [25]). The state-variables $x_{i}(t), i=1, \ldots, n$, in the RFM represent occupancy levels on a coarse-grained model of the mRNA, normalized so that $x_{i}(t) \in[0,1]$ for all $t$. The state-space of the RFM is thus $C^{n}:=[0,1]^{n}$. It is straightforward to show, using the results presented here, that the RFM is not contractive with respect to any norm on $C^{n}$, yet is ST on $C^{n}$.

Acknowledgements: We thank Zvi Artstein for helpful comments.

\section{REFERENCES}

[1] W. Lohmiller and J.-J. E. Slotine, "On contraction analysis for nonlinear systems," Automatica, vol. 34, pp. 683-696, 1998.
[2] G. Russo, M. di Bernardo, and E. D. Sontag, "Global entrainment of transcriptional systems to periodic inputs," PLoS Computational Biology, vol. 6, p. e1000739, 2010.

[3] W. Lohmiller and J.-J. E. Slotine, "Control system design for mechanical systems using contraction theory," IEEE Trans. Automat. Control, vol. 45, pp. 984-989, 2000.

[4] S. Bonnabel, A. Astolfi, and R. Sepulchre, "Contraction and observer design on cones," in Proc. 50th IEEE Conf. on Decision and Control and European Control Conference, Orlando, Florida, 2011, pp. 71477151.

[5] W. Wang and J. J. Slotine, "On partial contraction analysis for coupled nonlinear oscillators," Biol. Cybern., vol. 92, pp. 38-53, 2005.

[6] J.-J. E. Slotine, "Modular stability tools for distributed computation and control," Int. J. Adaptive Control and Signal Processing, vol. 17, pp. 397-416, 2003.

[7] G. Russo, M. di Bernardo, and E. Sontag, "A contraction approach to the hierarchical analysis and design of networked systems," IEEE Trans. Automat. Control, vol. 58, pp. 1328-1331, 2013.

[8] D. Angeli, "A Lyapunov approach to incremental stability properties," IEEE Trans. Automat. Control, vol. 47, pp. 410-421, 2002.

[9] F. Forni and R. Sepulchre, "A differential Lyapunov framework for contraction analysis," 2013. [Online]. Available: http://arxiv.org/abs/1208.2943v2

[10] Z. Aminzare and E. D. Sontag, "Logarithmic Lipschitz norms and diffusion-induced instability," Nonlinear Analysis: Theory, Methods \& Applications, vol. 83, pp. 31-49, 2013.

[11] Q.-C. Pham, N. Tabareau, and J.-J. Slotine, "A contraction theory approach to stochastic incremental stability," IEEE Trans. Automat. Control, vol. 54, pp. 816-820, 2009.

[12] G. Soderlind, "The logarithmic norm. History and modern theory," BIT Numerical Mathematics, vol. 46, pp. 631-652, 2006.

[13] J. Jouffroy, "Some ancestors of contraction analysis," in Proc. 44th IEEE Conf. on Decision and Control, Seville, Spain, 2005, pp. 54505455.

[14] B. S. Rüffer, N. van de Wouw, and M. Mueller, "Convergent systems vs. incremental stability," Systems Control Lett., vol. 62, pp. 277-285, 2013.

[15] M. Margaliot, E. D. Sontag, and T. Tuller, "Entrainment to periodic initiation and transition rates in a computational model for gene translation," PLOS ONE, vol. 9, no. 5, p. e96039, 2014.

[16] F. Dorfler and F. Bullo, "Synchronization and transient stability in power networks and nonuniform Kuramoto oscillators," SIAM J. Control Optim., vol. 50, pp. 1616-1642, 2012.

[17] H. L. Smith, Monotone Dynamical Systems: An Introduction to the Theory of Competitive and Cooperative Systems, ser. Mathematical Surveys and Monographs. Providence, RI: Amer. Math. Soc., 1995, vol. 41.

[18] E. D. Sontag, Mathematical Control Theory: Deterministic FiniteDimensional Systems, 2nd ed., ser. Texts in Applied Mathematics. New York: Springer-Verlag, 1998, vol. 6.

[19] D. Angeli and E. D. Sontag, "Monotone control systems," IEEE Trans. Automat. Control, vol. 48, pp. 1684-1698, 2003.

[20] M. Vidyasagar, Nonlinear Systems Analysis. Englewood Cliffs, NJ: Prentice Hall, 1978.

[21] S. Reuveni, I. Meilijson, M. Kupiec, E. Ruppin, and T. Tuller, "Genome-scale analysis of translation elongation with a ribosome flow model," PLoS Computational Biology, vol. 7, p. e1002127, 2011.

[22] M. Margaliot and T. Tuller, "Stability analysis of the ribosome flow model," IEEE/ACM Trans. Computational Biology and Bioinformatics, vol. 9, pp. 1545-1552, 2012.

[23] M. Margaliot and T. Tuller, "Ribosome flow model with positive feedback," J. Royal Society Interface, vol. 10, p. 20130267, 2013.

[24] M. Margaliot and T. Tuller, "On the steady-state distribution in the homogeneous ribosome flow model," IEEE/ACM Trans. Computational Biology and Bioinformatics, vol. 9, pp. 1724-1736, 2012.

[25] Y. Zarai, M. Margaliot, and T. Tuller, "Explicit expression for the steady state translation rate in the infinite-dimensional homogeneous ribosome flow model," IEEE/ACM Trans. Computational Biology and Bioinformatics, vol. 10, pp. 1322-1328, 2013. 